\title{
Specifying and Verbalising Answer Set Programs in Controlled Natural Language
}

\author{
ROLF SCHWITTER \\ Department of Computing, Macquarie University, Sydney, NSW 2109, Australia \\ (e-mail: Rolf.Schwitter@mq.edu.au)
}

submitted 28 April 2018; accepted 17 May 2018

\begin{abstract}
We show how a bi-directional grammar can be used to specify and verbalise answer set programs in controlled natural language. We start from a program specification in controlled natural language and translate this specification automatically into an executable answer set program. The resulting answer set program can be modified following certain naming conventions and the revised version of the program can then be verbalised in the same subset of natural language that was used as specification language. The bi-directional grammar is parametrised for processing and generation, deals with referring expressions, and exploits symmetries in the data structure of the grammar rules whenever these grammar rules need to be duplicated. We demonstrate that verbalisation requires sentence planning in order to aggregate similar structures with the aim to improve the readability of the generated specification. Without modifications, the generated specification is always semantically equivalent to the original one; our bi-directional grammar is the first one that allows for semantic round-tripping in the context of controlled natural language processing.
\end{abstract}

KEYWORDS: Bi-directional grammars, controlled natural languages, answer set programming, sentence planning, executable specifications

\section{Introduction}

There exist a number of controlled natural languages (Sowa 2004; Clark et al. 2005; Fuchs et al. 2008; Guy and Schwitter 2017) that have been designed as high-level interface languages to knowledge systems. However, none of the underlying grammars of these controlled natural languages is bi-directional in the sense that a specification can be written in controlled natural language, translated into a formal target representation, and then - after potential modifications of that target representation - back again into the same subset of natural language. This form of round-tripping under modification is difficult to achieve, in particular with a single grammar that can be used for processing as well as for generation. In this paper, we show how such a bi-directional grammar can be built in form of a logic program for a controlled natural language. We discuss the implementation of a definite clause grammar that translates a controlled natural language specification into an executable answer set program. The resulting answer set program can be modified and then verbalised in controlled natural language using the same grammar exploiting symmetries in the feature structures of the grammar rules. Without modification of the original answer set program, this leads to a form of round-tripping that preserves semantic equivalence but not syntactic equivalence. This work has certain similarities with our previous work (Schwitter 2008) where a bi-directional grammar is used to translate an experimental 
controlled natural language into syntactically annotated $\mathrm{TPTP}^{1}$ formulas; these annotated formulas are then stored and serve as templates to answer questions in controlled natural language. The novelty of the presented work is that no such annotated formulas are required; instead a sentence planner is used that applies different generation strategies. The definite clause grammar uses an internal format for the representation of clauses for the answer set program. This internal format is designed in such a way that it can be used for processing and generation purposes and it respects at the same time accessibility constraints of antecedents for referring expressions. The grammar of the controlled natural language for answer set programs is more restricted than a corresponding grammar for first-order formulas, since some linguistic constructions can only occur at certain positions in particular sentences. However, in contrast to a controlled natural language that is equivalent to first-order logic, a controlled language for answer set programs allows us to distinguish, for example, between strong and weak negation using two separate linguistic constructions for these forms of negation.

The rest of this paper is structured as follows: In Section 2, we recall linguistic preliminaries and sketch the controlled natural language to be used. In Section 3, we briefly review answer set programming and show how it has been employed for natural language processing, with a particular focus on controlled natural language processing. In Section 4, we present a specification written in controlled natural language, discuss the resulting answer set program and its verbalisation. In Section 5, we present the bi-directional grammar in detail and explain how symmetries in the feature structures of the grammar rules can be exploited to process and generate sentences. In Section 6, we introduce the sentence planner and a more complex specification, and discuss the planning strategies that are applied to the internal format for answer set programs before the bi-directional grammar is used to verbalise an answer set program. In Section 7, we summarise the key points of our approach, highlight its benefits, and conclude.

\section{Linguistic Preliminaries}

The controlled natural language that we discuss here is based on the one used in the PENG ${ }^{\text {ASP }}$ system (Guy and Schwitter 2017). This system translates specifications written in controlled language into answer set programs but not back again. This is because the grammar of the language PENG ${ }^{\text {ASP }}$ has not been designed with bi-directionality in mind. The language PENG ${ }^{A S P}$ distinguishes between simple and complex sentences. We focus in the following introduction mainly on those linguistic constructions that occur also in the subsequent example specifications.

\subsection{Simple Sentences}

Simple sentences in our controlled natural language consist of a subject and a predicate, and include zero or more complements depending on the verbal predicate. For example, the following are simple sentences:

1. Bob is a student.

2. Sue Miller works.

3. Tom studies at Macquarie University.

1 http://www.cs.miami.edu/ $/ \mathrm{tptp} /$ 
4. The Biology book is expensive.

5. There is a student.

Sentence (1) consists of a proper name $(B o b)$ in subject position, followed by a linking verb (is), and a subject complement (a student). Sentence (2) contains an intransitive verb (works) but no complement. Sentence (3) contains a transitive prepositional verb (studies at) with a complement in form of a proper name (Macquarie University) in object position. Sentence (4) consists of a definite noun phrase (The Biology book) in subject position followed by a linking verb and an adjective (expensive) as subject complement. Sentence (5) shows that it is possible to introduce new entities with an expletive there-construction where there acts as filler for the subject.

\subsection{Complex Sentences}

Complex sentences are built from simpler sentences through coordination, subordination, quantification, and negation:

6. Tom studies at Macquarie University and is enrolled in Information Technology.

7. Bob who is enrolled in Linguistics studies or parties.

8. Tom is enrolled in COMP329, COMP330 and COMP332.

9. Every student who works is successful.

10. It is not the case that a student who is enrolled in Information Technology parties.

11. If a student does not provably work then the student does not work.

In sentence (6), two verb phrases are coordinated with the help of a conjunction (and). In sentence (7), a subordinate relative clause (who is enrolled in Linguistics) is used in subject position and two verb phrases are coordinated with the help of a disjunction (or). Sentence (8) contains a coordination in form of an enumeration (COMP329, COMP330 and COMP332) in object position. Sentence (9) uses a universally quantified noun phrase (Every student) that contains a relative clause (who works) in subject positions. In (10), the entire complex sentence is negated with the help of the predefined construction It is not the case that; and finally in (11), weak negation (does not provably work) is used in the condition of a conditional sentence and strong negation (does not work) in the consequent of that sentence.

\section{Answer Set Programming and Natural Language Processing}

Answer set programming is a modern approach to declarative programming and has its roots in logic programming, logic-based knowledge representation and reasoning, deductive databases, and constraint solving (Gelfond and Lifschitz 1988; Baral 2003; Lifschitz 2008; Gebser et al. 2017). The main idea behind answer set programming is to represent a computational problem as a (non-monotonic) logic program whose resulting models (= answer sets) correspond to solutions of the problem. Answer set programs consist of clauses that look similar to clauses in Prolog; however, in answer set programming an entirely different computational mechanism is used that computes answer sets in a bottom-up fashion. An answer set program consists of a set of clauses (rules) of the form:

$$
\mathrm{L}_{1} ; \ldots ; \mathrm{L}_{k}:-\mathrm{L}_{k+1}, \ldots, \mathrm{L}_{m} \text {, not } \mathrm{L}_{m+1}, \ldots, \text { not } \mathrm{L}_{n} .
$$

where all $\mathrm{L}_{i}$ are literals or strongly negated literals. The connective ": -" stands for if and separates the head from the body of the rule. The connective ";" in the head of the rule stands for an 


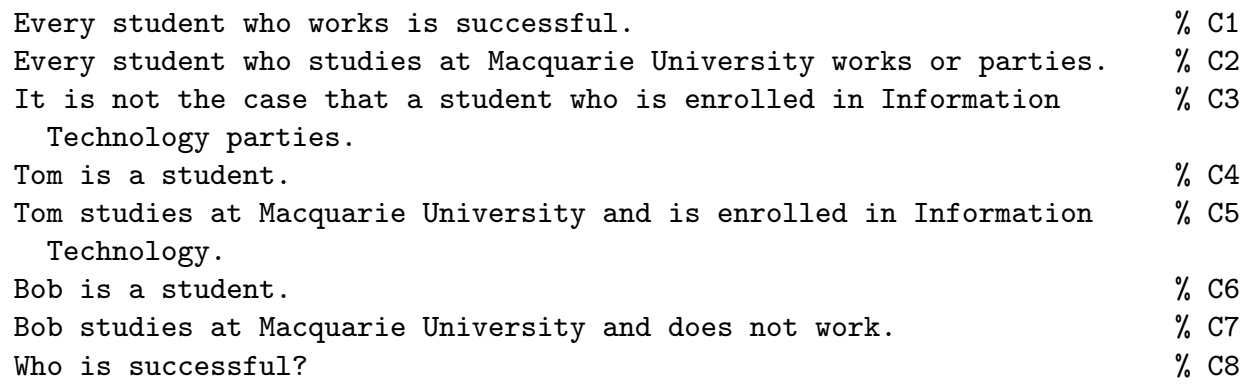

Fig. 1. Successful Student in Controlled Natural Language

epistemic disjunction (at least one literal is believed to be true). The connective "," in the body of the rule stands for and and the connective "not" for negation as failure (also called weak negation). A rule with an empty body and without an if-connective is called a fact, and a rule without a head is called a constraint. Answer set programming is supported by powerful tools; for example, the clingo system (Gebser et al. 2017) combines a grounder and a solver. The grounder converts an answer set program that contains variables into an equivalent ground (variable-free) propositional program and the solver computes the answer sets of the propositional program. Answers to questions can then be extracted directly from the resulting answer sets.

Answer set programming is an interesting formalism for knowledge representation in the context of natural language processing because of its ability to combine strong and weak negation which offers support for non-monotonic reasoning. Answer set programming has been used for syntactic parsing in the context of the Combinatory Categorial Grammar formalism (Lieler and Schüller 2012; Schüller 2013), for semantic parsing and representation of textual information (Baral et al. 2011; Nguyen et al. 2015), and for natural language understanding in the context of question answering (Tari and Baral 2005) and the Winograd Schema Challenge (Schüller 2014; Bailey et al. 2015). In the context of controlled natural language processing, answer set programming has been used as a prototype of a rule system in the Attempto project (Kuhn 2007; Fuchs et al. 2008), as a target language for biomedical queries related to drug discovery (Erdem and Yeniterzi 2009), as a source language for generating explanations for biomedical queries (Erdem and Öztok 2015), as a framework for human-robot interaction (Demirel et al. 2016), and as a target language for writing executable specifications (Guy and Schwitter 2017).

\section{From Controlled Natural Language to Answer Set Programs and Back Again}

Before we discuss the implementation of our bi-directional grammar for answer set programs in Section 5, we first illustrate how our controlled natural language can be used for specifying and verbalising answer set programs, and thereby motivate its usefulness.

Figure 1 shows a specification written in controlled natural language. This specification consists of two universally quantified sentences ( $\mathrm{C} 1$ and $\mathrm{C} 2$ ), a constraint (C3), four declarative sentences (C4-C7), followed by a wh-question (C8). Note that the two universally quantified sentences contain an embedded relative clause in subject position; the second one of these universally quantified sentences (C2) contains a disjunctive verb phrase. The two declarative sentences ( $\mathrm{C5}$ and $\mathrm{C7}$ ) are complex and contain coordinated verb phrases. Note also that the 


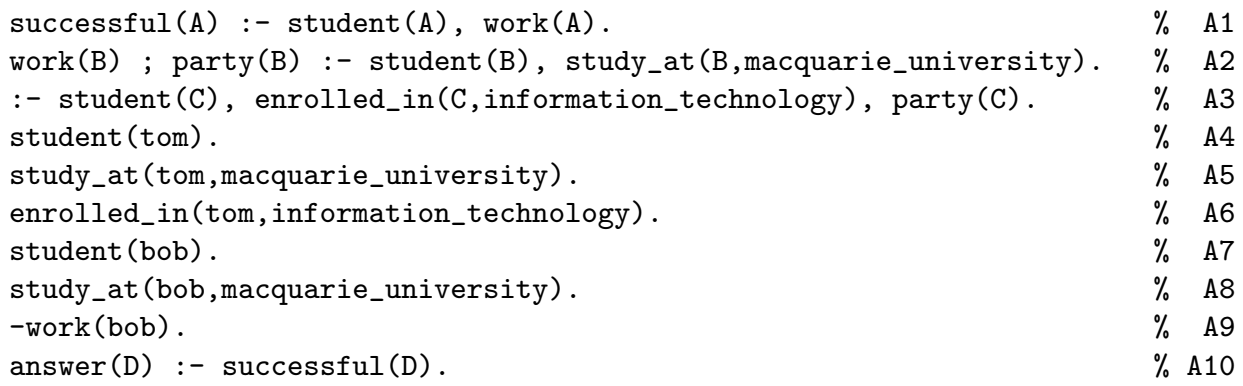

Fig. 2. Successful Student as Answer Set Program

proper name Macquarie University in C5 and C7 is used anaphorically and links back to the proper name Macquarie University introduced in $\mathrm{C} 2$. This specification is automatically translated into the answer set program displayed in Figure 2.

Note that the translation of sentence $\mathrm{C} 1$ results in a normal clause (A1) with a single literal in its head. The translation of sentence $\mathrm{C} 2$ leads to a clause with a disjunction in its head (A2), and the translation of sentence $\mathrm{C} 3$ to a constraint (A3). The translation of the two declarative sentences $\mathrm{C} 4$ and $\mathrm{C} 6$ results in a single fact $(\mathrm{A} 4+\mathrm{A} 7)$ for both sentences, and the translation of the two coordinated sentences $\mathrm{C} 5$ and $\mathrm{C} 7$ leads to two facts each (A5+A6 and A8+A9). In the case of $\mathrm{C} 7$ one of these facts is strongly negated (A9). Finally, the translation of the wh-question C8 results in a rule with a specific answer literal (answer(D)) in its head (A10). The user can now modify this answer set program as illustrated in Figure 3 - as long as they stick to the existing naming convention.

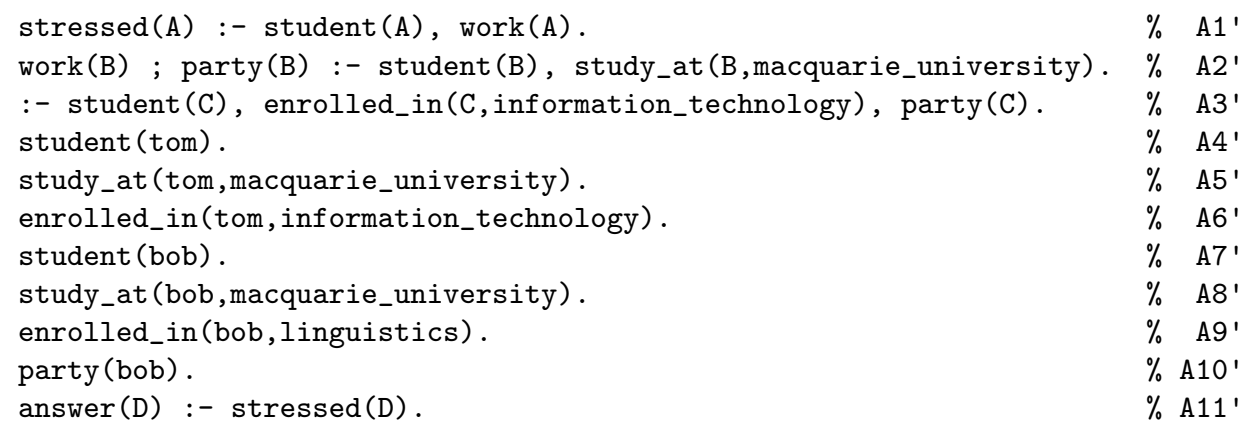

Fig. 3. Modified Answer Set Program

This modified answer set program contains the following changes: the literal successful (A) in the head of the clause A1 has been replaced by the literal stressed(A) on line A1 '; the new fact enrolled_in(bob,linguistics) has been added to the program on line A9'; the negated literal -work (bob) on line A9 has been replaced by the positive literal party (bob) on line A10 '; and the literal successful(D) in the body of the clause that answers the question on line $\mathrm{A} 10$ has been replaced by the literal stressed(D) on line A11'. This modified answer set program can now be automatically verbalised in controlled natural language as illustrated in Figure 4. 


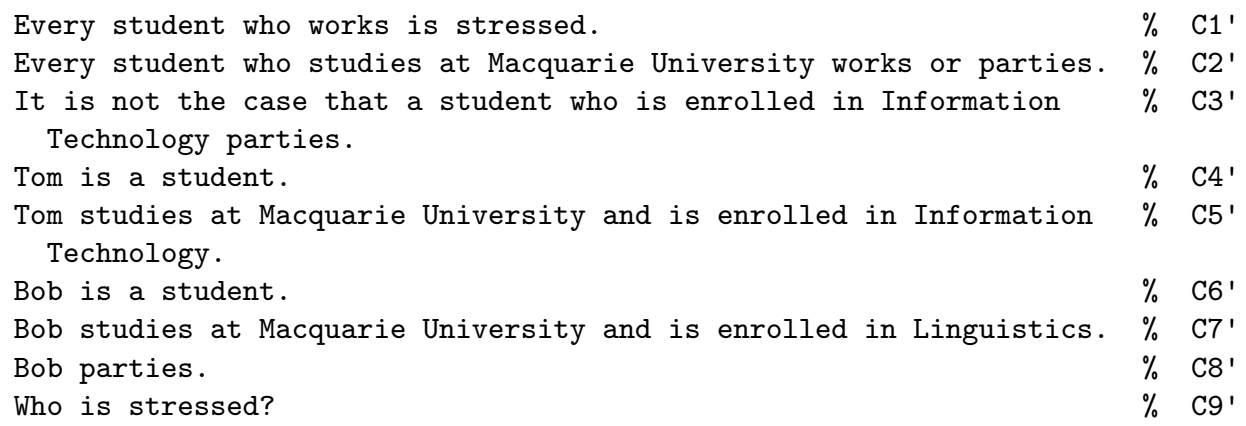

Fig. 4. Verbalisation of the Modified Answer Set Program in Controlled Natural Language

We observe that the clauses on line $\mathrm{A5}$ ' and $\mathrm{A6}$ ' and similarly those on line $\mathrm{A} 8^{\prime}$ and $\mathrm{A} 9$ ' of the modified answer set program have been used to generate a coordinated verb phrase as shown on line $\mathrm{C} 5$ ' and $\mathrm{C} 7$ ' of Figure 4. This is a form of aggregation that has been executed by the sentence planner and combines similar structures that fall under the same subject based on the proximity of the clauses. Alternatively, the sentence planner could use another aggregation strategy and combine, for example, the clauses on line $\mathrm{A} 4$ ' and $\mathrm{A} 5$ ' or those on line $\mathrm{A} 4$ ' and $\mathrm{A} 5$ ' and $\mathrm{A} 6$ ' into a verb phrase (more on this in Section 6).

\section{Implementation of the Bi-directional Grammar}

To implement our bi-directional grammar, we use the definite clause grammar (DCG) formalism (Pereira and Warren 1980; Pereira and Shieber 1987). This formalism gives us a convenient notation to express feature structures and the resulting grammar can easily be transformed into another notation via term expansion, if an alternative parsing strategy is required (Guy and Schwitter 2017). Our bi-directional grammar implements a syntactic-semantic interface where the meaning of a textual specification and its linguistic constituents is established in a compositional way during the translation of the specification into an answer set program. Unification is used to map the syntactic constituents of the controlled language on terms and (partial) clauses of the corresponding answer set program. In our context, a textual specification $A$ and its verbalisation $A^{\prime}$ can be shown to be semantically equivalent, if they both generate the same answer set program that entails the same solutions.

Our implementation of the grammar relies on four other components that are necessary to build an operational system: (1) a tokeniser that splits a specification written in controlled natural language into a list of tokens for each sentence; (2) a writer that translates the internal format constructed during the parsing process into the final answer set program; (3) a reader that reads a (potentially modified) answer set program and converts this program into the internal format; and (4) a sentence planner that tries to apply a number of aggregation strategies, reorganises the internal format for this purpose, before it is used by the grammar for the generation process.

\subsection{Internal Format for Answer Set Programs}

The bi-directional definite clause grammar uses an internal format for answer set programs and a special feature structure that distinguishes between a processing and a generation mode in the 
grammar rules. Depending on this mode, the same difference list (Sterling and Shapiro 1994) is used as a data structure to either construct the internal format for an input text or to deconstruct the internal format to generate a verbalisation. The internal format is based on a flat notation that uses a small number of typed predicates (e.g. class, pred, prop, named) that are associated with linguistic categories (e.g. noun, verb, adjective, proper name) in the lexicon. This flat notation allows us to abstract over individual predicate names and makes the processing for the anaphora resolution algorithm and sentence planner easier. Let us briefly illustrate the form and structure of this internal format with the help of the following two example sentences:

12. Tom is a student and works.

13. If a student works then the student is successful.

In the case of processing, the tokeniser sends a list of tokens for these two sentences to the bi-directional grammar that builds up the following internal format during the parsing process with the help of the information that is available in the lexicon for these tokens:

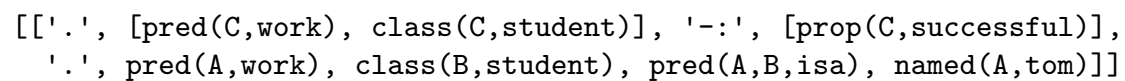

As this example shows, the internal format is built up in reverse order; the literals derived from the first sentence (12) follow the literals derived from the second sentence (13). As a consequence of this ordering, the arrow operator for the rule (-:) points from the body on the left side of the rule to the head on the right side. Note that this internal format retains the full stops in order to separate the representation of individual clauses and uses, in our example, two sublists as part of the second clause. These sublists constrain the accessibility of antecedents for referring expressions - in a similar way as postulated in discourse representation theory (Kamp and Reyle 1993; Geurts et al. 2015). This internal format is then automatically translated by the writer into the final answer set program. In order to achieve this, the writer reverses the order of the clauses, removes auxiliary literals, and turns the typed representation of literals into an untyped one:

student (tom). work (tom).

successful(C) :- student(C), work(C).

In the case of generation, the clauses of the answer set program are first read by the reader that reconstructs the flat internal format. Since we use the same data structure (difference list) in the grammar for processing and generation, the internal format for clauses is now built up in normal order and not in reverse order as before. This has - among other things - the effect that the arrow operator (:-) of the rule now points from the body of the rule on the right side to the head on the left side:

[ [named (A,tom), pred (A,B,isa), class (B, student), pred(B,work), '.', [prop(C, successful)], ':-', [class (C, student), pred(C,work)], '.']]

This reconstructed internal format is then sent to the sentence planner that tries to apply a number of strategies to aggregate the information in order to generate, for example, coordinated sentences or to take care of enumerations in sentences. As we will see in Section 6, sentence planning requires reorganising the internal format to achieve the intended result.

\subsection{Overview of Grammar Rules}

In this section we introduce the format of the bi-directional definite clause grammar rules. Since our grammar is a grammar for a controlled natural natural, specific restrictions apply to the 
grammar rules. We distinguish between grammar rules for declarative sentences, conditional sentences, constraints, and questions. This distinction is important since only certain syntactic constructions can occur at certain positions in these sentences. For example, a noun phrase in subject position of a declarative sentence can only be coordinated if this noun phrase is followed by a linking verb or a noun phrase that occurs in the subject position in the consequent of a conditional sentence can only have the form of a definite noun phrase. On the interface level, these syntactic constraints are enforced by a lookahead text editor that guides the writing process of the user and uses the grammar as starting point to extract the lookahead information (for details about this interface technology see (Guy and Schwitter 2017)).

When a specification is processed, the text is split up by the tokeniser into a list of lists where each list contains those tokens that represent a sentence. At the beginning the difference list that constructs the internal representation for an answer set program has the following form: [[]]-C, where the incoming part consists of a list that contains an empty list and the outgoing part consists of a variable; the same applies to the difference list that collects all accessible antecedents during the parsing process. When an answer set program is verbalised, the incoming part of the difference list consists of an embedded list of elements that stand for the internal format of the answer set program; of course, the lists for the tokens and accessible antecedents are empty at this point. It is important to note that these difference lists are processed recursively for each sentence or for each clause of an answer set program and that they establish a context for the actual interpretation.

The following is a grammar rule in definite clause grammar format; it takes as input either a list of tokens of a declarative sentence and updates the answer set program under construction or it takes as input a (partial) answer set program and produces a list of tokens that correspond to a declarative sentence:

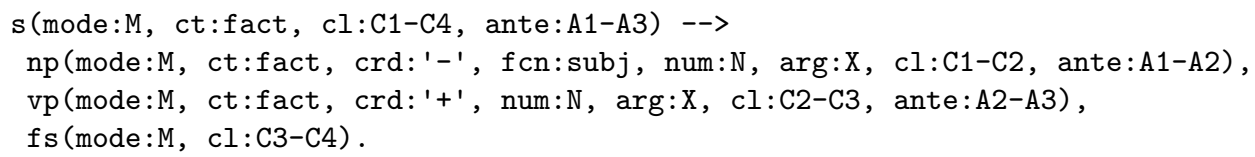

This grammar rule states that a declarative sentence ( $\mathrm{s}$ ) consists of a noun phrase (np) and a verb phrase (vp), followed by a full stop (fs). This grammar rule contains additional arguments that implement feature structures in the form of attribute:value pairs that can be directly processed via unification. The feature structure mode:M distinguishes between the processing mode (proc) and generation mode (gen). The feature structure ct:fact stands for the clause type and specifies that the noun phrase as well as the verb phrase of this rule are used to generate or process facts. The feature structures $\mathrm{cl}: \mathrm{C} 1-\mathrm{C} 4, \mathrm{cl}: \mathrm{C} 1-\mathrm{C} 2, \mathrm{cl}: \mathrm{C} 2-\mathrm{C} 3$, and $\mathrm{cl}: \mathrm{C} 3-\mathrm{C} 4$ implement a difference list and are used to construct the internal format for an answer set program or to deconstruct the internal format of an answer set program to drive the generation of a verbalisation. The feature structures ante:A1-A3, ante:A1-A2, and ante:A2-A3 implement a second difference list that stores all accessible antecedents. These accessible literals are continuously updated by the anaphora resolution algorithm during the parsing process. The feature structure crd: ' - ' specifies that the noun phrase of this rule cannot be coordinated and the feature structure crd: ' +' that the verb phrase of this rule can be coordinated. The feature structure num: $\mathrm{N}$ deals with number agreement between noun phrase and verb phrase, while the feature structure arg:X makes the variable for the entity denoted by the noun phrase in subject position ( $f \mathrm{cn}: \mathrm{subj}$ ) available for the literal described by the verb phrase. Our implementation uses additional feature structures that 
build up a syntax tree and a paraphrase during the parsing process but these feature structures are not shown in our example to keep the presentation clear and compact.

The following grammar rule is a preterminal rule that is used in the processing mode (proc) and adds the literal named ( $\mathrm{X}, \mathrm{Name}$ ) for a proper name to the outgoing part of the difference list that constructs the internal format and to the difference list that stores all accessible antecedents:

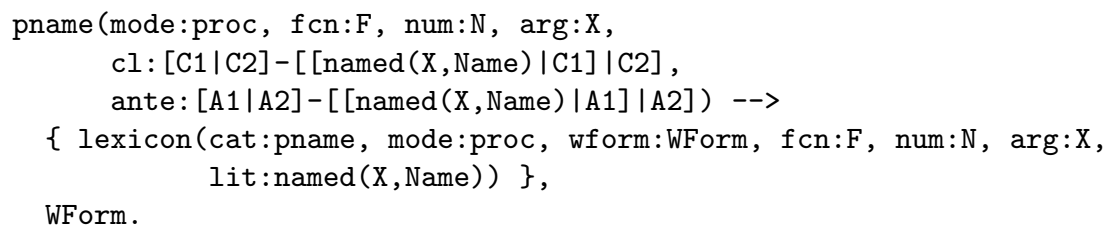

Note that the outgoing list contains all information of the incoming list plus the added literal. At this point, this addition is only preliminary and the anaphora resolution algorithm will decide if this literal is new or not. The grammar rule that is used in the generation mode (gen) for proper names looks very similar to the grammar rule in the processing mode, but now a literal for the proper name is removed from the incoming list of clauses instead of added to the outgoing list:

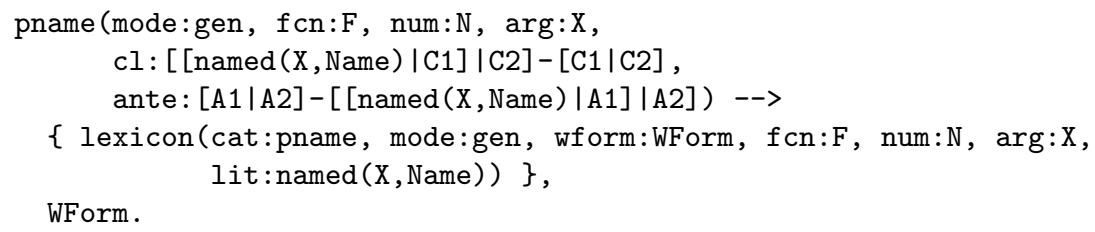

The grammar rule below translates conditional sentences into the internal format for rules and the internal format for rules into conditional sentences:

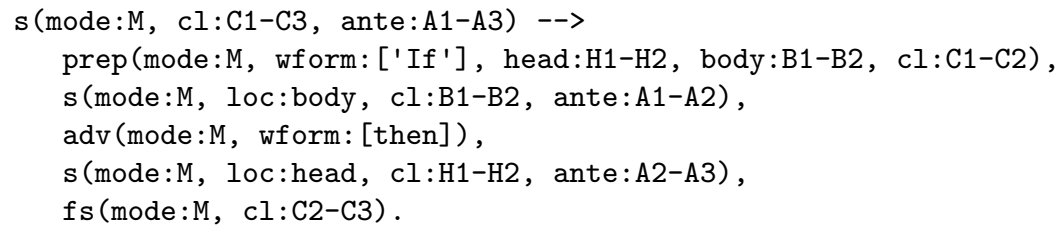

The feature structures $\mathrm{cl}: \mathrm{C} 1-\mathrm{C} 3, \mathrm{cl}: \mathrm{C} 1-\mathrm{C} 2$, and $\mathrm{cl}: \mathrm{C} 2-\mathrm{C} 3$ implement again a difference list and are used to construct or deconstruct the internal format of a rule. The two feature structures $\mathrm{cl}: \mathrm{H} 1-\mathrm{H} 2$ and $\mathrm{cl}: \mathrm{B} 1-\mathrm{B} 2$ implement another difference list that provides the relevant data structures for the head and body of the rule. The feature structures ante:A1-A3, ante:A1-A2, and ante:A2-A3 are used as before to store those antecedents that are currently accessible. The overall structure for the rule as well as the structure of the head and the body for the rule become available via the following preterminal grammar rules:

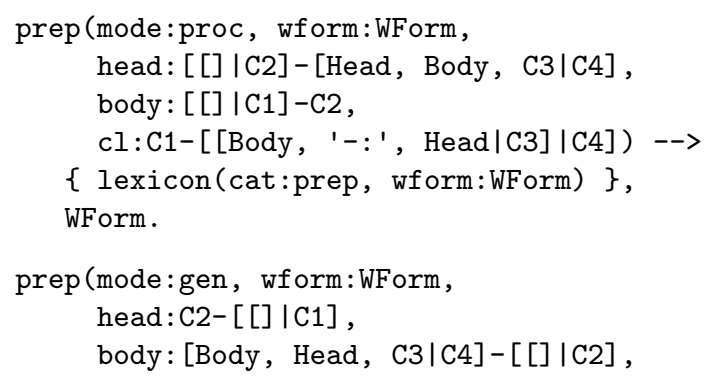




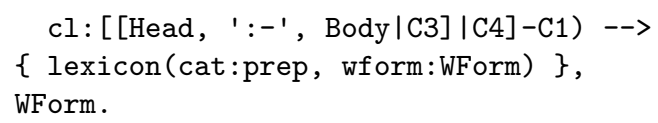

The first rule is used for processing and the second one for generation. In the case of processing a conditional sentence, first the body of a rule is constructed and then the head. Constructing the body of a rule starts with an empty list ([]) and takes the already processed context in list $\mathrm{C} 1$ into consideration for anaphora resolution. During the parsing process literals are added to the empty list, and the resulting outgoing list (C2) then builds the new context for processing the head of the rule. The processing of the head of the rule starts again with an empty list and results in an outgoing list that consists of a list (Head) that contains the head literals and a list (Body) that contains the body literals. Note that the variable C3 stands for a list that contains the previously processed context. These elements are then combined in a rule in the outgoing part of the difference list ([ [Body, '-: ', Head/C3] IC4]) that build up the clauses for the answer set program. It is interesting to see that we end up in the case of generation with feature structures that are symmetric to processing. In the case of generation, a rule is deconstructed into a body (Body) and a head (Head), and then the body is first processed followed by the head. Note that the successful processing of the body results in an empty list ([]) in the outgoing difference list, the same happens after the processing of the head. We observe that all grammar rules for preterminal symbols are symmetric, including grammar rules that process cardinality quantifiers (cqnt) such as exactly Number Noun, at least Number Noun, and at most Number Noun:

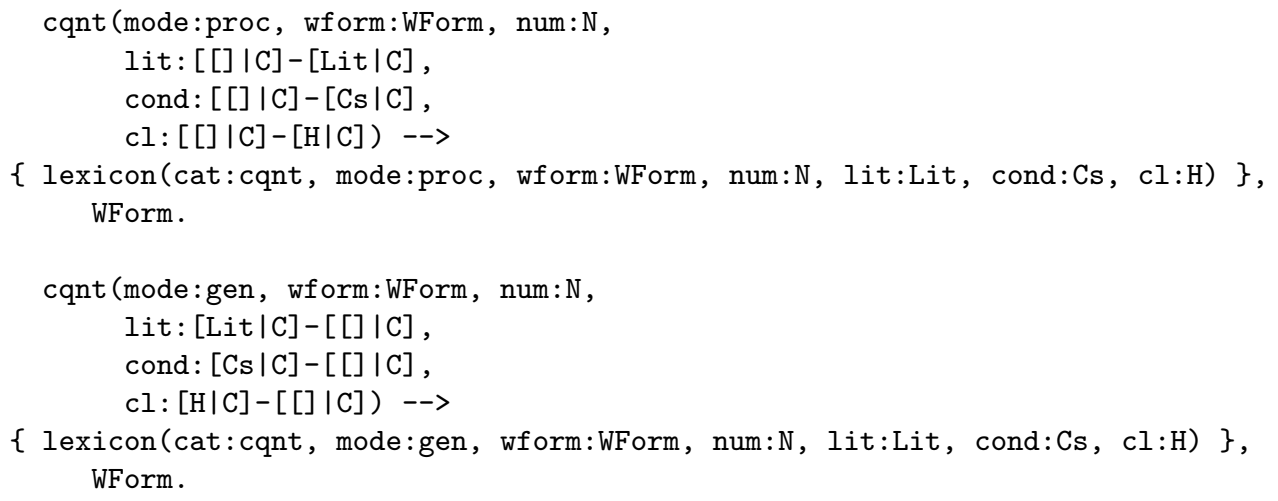

The two rules above take part in the processing and generation of clauses with a cardinality constraint in the head: Lower 1 Lit:Ds $\}$ Upper $_{2}:-$ BODY. Here the expression Lit:Ds is a conditional literal consisting of a basic literal (Lit) and one or more domain predicates (Ds).

\subsection{Anaphora Resolution}

Proper names and definite noun phrases can be used as referring expressions in the controlled natural language $\mathrm{PENG}^{\mathrm{ASP}}$. In our case the list structure of the internal representation constrains the accessibility of antecedents for referring expressions. In particular, a referring expression can only refer to an expression in the current list or to an expression in a list superordinate to that list. A list that encloses the current list is superordinate to it; and in addition, a list in the body of a rule is superordinate to the list in the head of the rule, but not vice versa.

The following grammar rule processes a definite noun phrase and checks whether this definite noun phrase introduces a new entity or whether it is used anaphorically. After processing the 
determiner (det) and the noun (noun), the grammar rule calls the anaphora resolution algorithm for definite noun phrases (def) and updates the internal format:

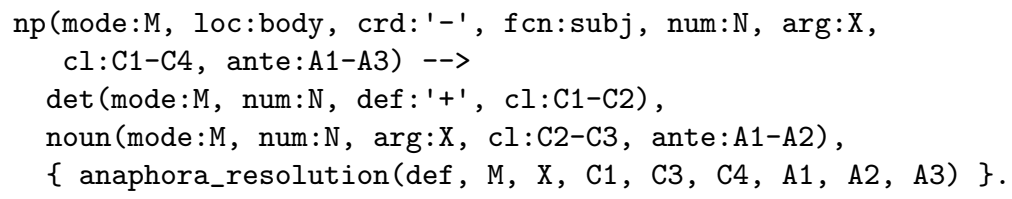

That means after processing of a definite noun phrase and temporarily adding the corresponding literal (for example, class (X, student)) to the outgoing part (C3) of the difference list, the anaphora resolution algorithm checks, whether an accessible literal already exists in the data structure $\mathrm{C} 1$ or not. If one exists, then the variable (x) of the newly added literal is unified with the variable of the closest accessible antecedent and the temporarily added literal is removed from the outgoing part of the difference list C3; resulting in C4. If no accessible antecedent exists, then the definite noun phrase is treated as an indefinite one and the new literal is not removed. The anaphora resolution algorithm also updates the list of accessible antecedents (A3) at the same time that can then be used to generate a list of referring expressions for the user interface.

The same grammar rule is used in the case of generation to decide whether a definite noun phrase can be generated or not. Since the internal data structure is reduced during the generation process, the anaphora resolution algorithm uses the list of accessible antecedents (A1) that is built up in parallel to check if a definite noun phrase can be generated or not.

\section{Sentence Planner}

In the case of generation, the reader first reads the answer set program and reconstructs the internal format. The sentence planner then tries to apply a number of aggregation strategies (Reiter and Dale 2000; Horacek 2015). Aggregation requires to reorganise the internal format of the answer set program. Remember that the internal format relies on a flat notation. In order to reconstruct this internal format for the modified answer set program in Figure 3, the reader has to add literals of the form named (Var, Name) to the internal representation for each constant (proper name) that occurs in the answer set program. After this reconstruction process, the result looks, for example, as follows for $\mathrm{A} 5$ ' and $\mathrm{A} 6$ ':

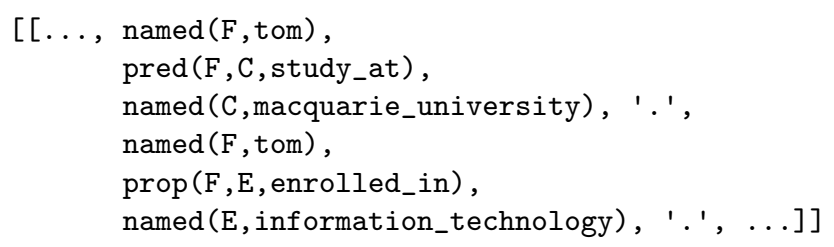

In the next step, the sentence planner tries to apply a suitable aggregation strategy using this reconstructed representation as a starting point. One possible strategy is to identify identical literals that can occur in the subject position (for example, named (F, tom)) and literals with the same number of arguments (for example, pred (F,C, study_at) and prop (F,E, enrolled_in)) that can occur in the predicate position, and aggregate these literals in order to generate a coordinated verb phrase. In our case, the sentence planner generates the following internal structure for verb phrase coordination: 


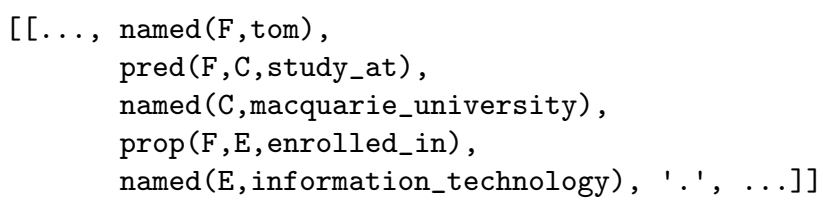

The sentence planner only aggregates up to three literals in predicate position and takes the number of their arguments and their proximity into consideration. That means no more than three verbs/relational adjectives with the same number of complements are currently coordinated in order to achieve good readability of the resulting specification.

In many cases the reconstruction of the internal structure is more complex and the sentence planner needs to select among the available strategies. We illustrate why this is the case with the help of the graph colouring problem (Gebser et al. 2012). The graph colouring problem deals with the assignment of different colours to nodes of a graph under certain constraints as specified in controlled natural language in Figure 5:

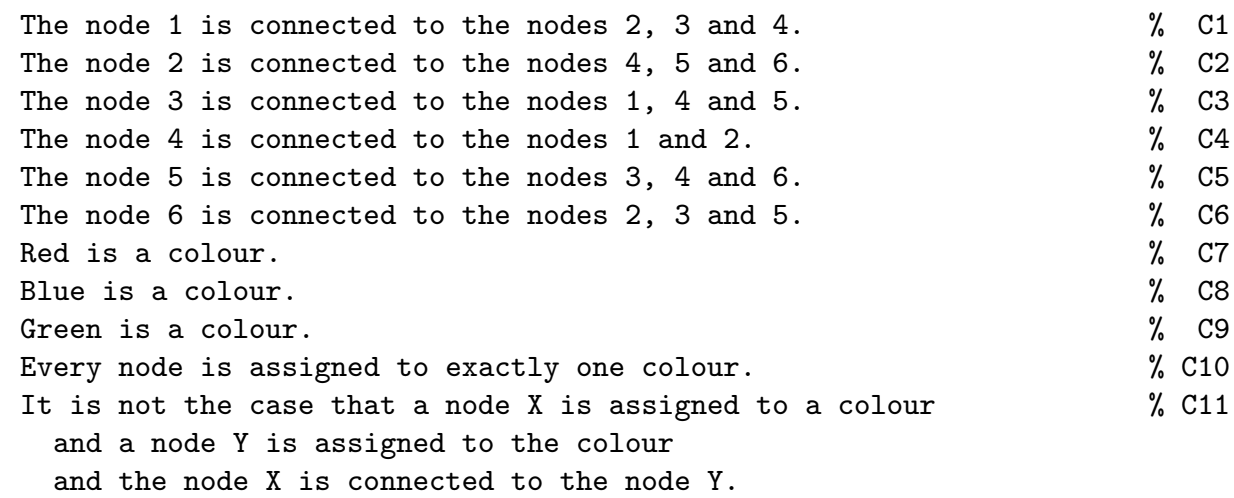

Fig. 5. Graph Colouring Problem in Controlled Natural Language

This specification shows the use of enumerations in object position (C1-C6). It also uses explicit variables ( $\mathrm{X}$ and $\mathrm{Y}$ ) in $\mathrm{C} 11$ to distinguish noun phrases that have the same form but denote different entities. The translation of this specification results in the answer set program in Figure 6 . This program does not keep all the information that is available in the internal format. In the internal format, integers (1-6) are represented with the help of literals of the form integer (Var, Integer) but result in integer arguments (for example, node(1)) in the answer set program and variables (X and $\mathrm{Y}$ ) are represented via literals of the form variable (Var, VariableName) which are not necessary anymore in the answer set program after anaphora resolution.

Generating a verbalisation that starts from the answer set program in Figure 6 requires again first the explicit reconstruction of the internal representation by the reader. For example, this reconstruction looks as follows for $\mathrm{A} 4 \mathrm{~A}$ ':

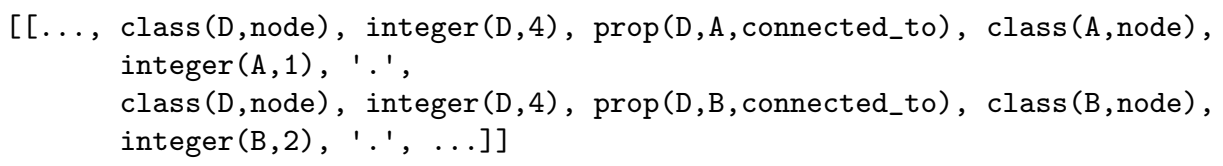




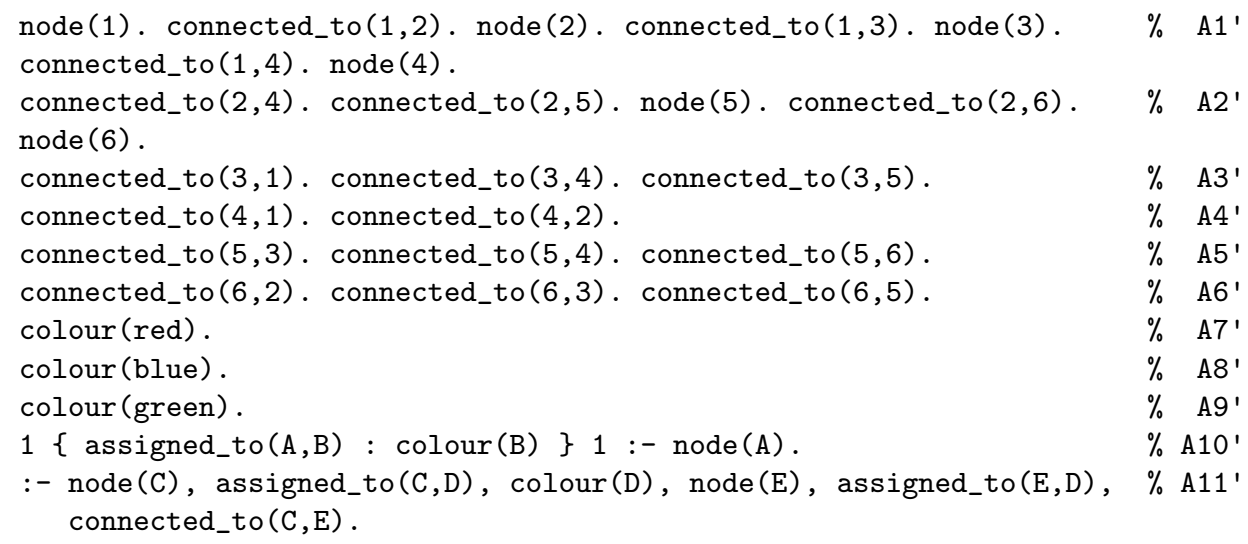

Fig. 6. Graph Colouring Problem as Answer Set Program

The sentence planner then reorganises this internal format in order to prepare for enumerating the integers $(1,2)$ of those nodes in object position that have the same subject and the same property name. In our case, this results in the following internal representation:

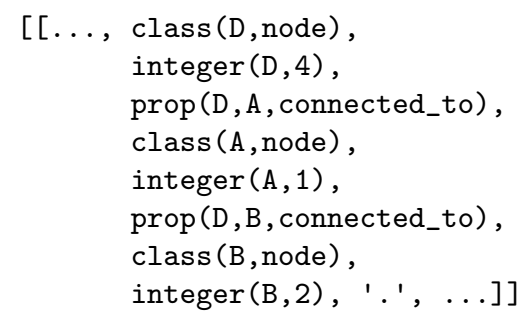

The grammar rules are ordered in such a way that priority is given to enumeration for this structure and not to verb phrase coordination. In our case, the original sentence $\mathrm{c} 4$, here repeated as (a), is generated and not the sentence (b):

a. The node 4 is connected to the nodes 1 and 2 .

b. The node 4 is connected to the node 1 and is connected to the node 2 .

In the case of $\mathrm{A} 11^{\prime}$, the reconstruction process is more complex since the clause in the answer set program contains two literals (node (C) and node(E)) with the same name. These literals need to be distinguished on the level of the controlled natural language with the help of variable names. That means literals of the form variable ( Var, VariableName) that represent variable names need to be added to the internal representation:

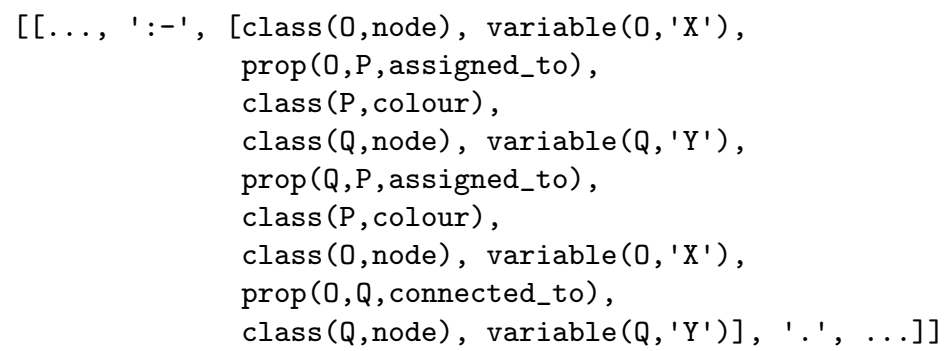


In order to generate a verbalisation that distinguishes between indefinite and definite noun phrases in the same way as illustrated in $\mathrm{C} 11$ of Figure 5, the first occurrence of the literals that generate an indefinite noun phrase (for example, class $\left(0\right.$, node), variable $\left(0, X^{\prime}\right)$ ) are added to the outgoing difference list that maintains all accessible antecedents, so that this information can be looked up later from that list to decide whether a definite noun phrase can be generated or not if the same literals occur a second time in the internal representation.

\section{Conclusion}

In this paper, we presented a bi-directional grammar for the controlled natural language PENG ${ }^{\mathrm{ASP}}$. This grammar uses the definite clause grammar formalism and allows us to translate a specification written in controlled natural language into an answer set program using a difference list as main data structure. This answer set program can then be translated (after potential modifications) back again into a controlled language specification. The same grammar rules can be used for processing and for generation; only those grammar rules that process preterminal symbols and add literals to or remove literals from the difference list need to be duplicated. However, these grammar rules have a nice symmetric structure that reflects the processing and generation task. The grammar also deals with referring expressions and resolves these expressions during the parsing process. The generation process requires sentence planning and allows us to generate semantically equivalent specifications if the answer set program has not been modified. If the program has been modified, then round-tripping is still possible as long as we stick to the same naming convention. The presented approach allows domain specialists who may not be familiar with answer set programming to specify and inspect an answer set program on the level of a controlled natural language. To the best of our knowledge, this is the first bi-directional grammar of a controlled natural language that can be used to process and verbalise answer set programs.

\section{References}

Bailey, D., Harrison, A., Lierler, Y., Lifschitz, V. And Michael, J. 2015. The Winograd Schema Challenge and Reasoning about Correlation. Working Notes of the Symposium on Logical Formalizations of Commonsense Reasoning.

Baral, C. 2003. Knowledge Representation, Reasoning and Declarative Problem Solving. Cambridge University Press.

Baral, C., DzifcaK, J., Gonzalez, M. A. And Zhou, J. 2011. Using Inverse $\lambda$ and Generalization to Translate English to Formal Languages. Proceedings of the Ninth International Conference in Computational Semantics, 35-44.

Clark, P., Harrison, P., Jenkins, T., Thompson, J. And Wojcik, R. 2005. Acquiring and Using World Knowledge using a Restricted Subset of English. The 18th International FLAIRS Conference (FLAIRS'05), 506-511.

Demirel, E., Gur, K. D., AND ERdem, E. 2016. Human-Robot Interaction in a Shopping Mall: A CNL Approach. CNL 2016, 111-122.

ERDEM, E. AND YENITERZI, R. 2009. Transforming controlled natural language biomedical queries into answer set programs. Proceedings of the Workshop on BioNLP, 117-124.

ERdem, E. AND ÖZToK, U. 2015. Generating explanations for biomedical queries. Theory and Practice of Logic Programming, Volume 15, Issue 1, 35-78.

Fuchs, N. E., Kaljurand, K. And Kuhn, T. 2008. Attempto Controlled English for Knowledge Representation. Reasoning Web, Fourth International Summer School 2008, LNCS 5224, 104-124. 
Gebser, M., Kaminski, R., Kaufmann, B. And Schaub, T. 2012. Answer Set Solving in Practice, Synthesis Lectures on Artificial Intelligence and Machine Learning, Morgan \& Claypool.

Gebser, M., Kaminski, R., Kaufmann, B., Lindauer, M., Ostrowski, M., Romero, J., Schaub, T. And Thiele, S. 2017. Potassco User Guide, Version 2.1.0, available at: https://github.com/potassco/guide/releases/, October 2017.

Gelfond, M. AND Lifschitz, V. 1988. The Stable Model Semantics for Logic Programming. Proceedings of the Fifth International Conference on Logic Programming (ICLP), 1070-1080.

Geurts, B., Beaver, D. I. And Maier, E. 2015. Discourse Representation Theory. Stanford Encyclopedia of Philosophy, available at: https://plato.stanford.edu/entries/discourserepresentation-theory/, 24th December, 2015.

GuY, S. ANd SchwitTer, R. 2017. The PENG ${ }^{\text {ASP }}$ System: Architecture, Language and Authoring Tool. Journal of Language Resources and Evaluation, Special Issue: Controlled Natural Language, Vol. 51, Issue 1, 67-92.

Horacek, H. 2015. New Concepts in Natural Language Generation: Planning, Realization and Systems. Linguistic Communication in Artificial Intelligence, Bloomsbury.

Kamp, H. And ReYle, U. 1993. From Discourse to Logic. Kluwer, Dordrecht.

Kunn, T. 2007. AceRules: Executing Rules in Controlled Natural Language. Proceedings of the First International Conference on Web Reasoning and Rule Systems (RR2007), LNCS, 299-308.

LIERLER, Y. AND SCHÜLleR, P. 2012. Parsing Combinatory Categorial Grammar via Planning in Answer Set Programming. Correct Reasoning, Springer, 436-453.

LifschitZ, V. 2008. What is Answer Set Programming? Proceedings of AAAI, 1594-1597.

Nguyen, H. V., Mitra, A. And BARAL, C. 2015. The NL2KR Platform for building Natural Language Translation Systems. Proceedings of ACL, 899-908.

Pereira, F. C. N. And WARRen, D. H. D. 1980. Definite Clause Grammars for Language Analysis - A Survey of the Formalism and a Comparison with Augmented Transition Networks. Artificial Intelligence, No. 13, 231-278.

Pereira, F. C. N. And Shieber, S. M. 1987. Prolog and Natural-Language Analysis. Cambridge University Press.

ReIter, E. AND DALE, R. 2000. Building Natural Language Generation Systems. Studies in Natural Language Processing. Cambridge University Press.

SCHÜLLER, P. 2013. Flexible Combinatory Categorial Grammar using the CYK Algorithm and Answer Set Programming. LPNMR, 499-511.

SCHÜLLER, P. 2014. Tackling Winograd Schemas by Formalizing Relevance Theory in Knowledge Graphs. International Conference on the Principles of Knowledge Representation and Reasoning (KR), 358-367.

SCHwitTER, R. 2008. Working for Two: a Bidirectional Grammar for a Controlled Natural Language. Proceedings of AI 2008, LNAI 5360, 168-179.

SowA, J. F. 2004. Common Logic Controlled English. Draft, available at: http://www.jfsowa. com/clce/specs.htm, 24 February 2004.

Sterling, L. S. And Shapiro, E. Y. 1994. The Art of Prolog, Advanced Programming Techniques. Second Edition, MIT Press.

TARI, L. AND BARAL C. 2005. Using AnsProlog with Link Grammar and WordNet for QA with deep reasoning. AAAI Spring Symposium Workshop on Inference for Textual Question Answering. 